\title{
INTERPRETAR, TRADUCIR, COMUNICAR: PAHSCH BASTEL VON DER SOHLE, MEDIADOR CULTURAL
}

Las más modernas teorías de la traducción, que renuncian al enfoque lingüístico-mecánico, se distancian cada vez más del concepto de mímesis en relación con la tarea o el arte de la traducción para fijarse en el traductor como mediador o intermediario cultural, cuando no como co-autor. Desde este punto de vista, se está replanteando no sólo la discusión teórico-metodológica sobre la traducción, sino analizando también la historia de la misma, aún incipiente en términos generales. Así, lenguaje, cultura, sociedad y, a veces política, entendida esta como hechos y acciones de orden supraindividual, nacional a veces, que inciden, sin embargo, de forma determinante en la cotidianidad particular, aparece cada vez más como un conjunto amalgamado en el que los conceptos de comunicación, comunidad y, de forma subsidiaria a la vez que superpuesta, de nación sirven de conectores en una relación de intercambio permanente.

Este ángulo se ofrece sobre todo para intentar comprender y analizar motivos, intenciones y procedimientos de traducción en relación con el surgimiento de las literaturas nacionales y la creación misma de los Estados modernos. Antes comunidad que nación, lengua que cultura, el caso alemán parece objeto especialmente adecuado de estudio, máxime cuando desde un momento muy temprano está presente Cervantes como referencia (más que) literaria ${ }^{1}$.

1 Hoy contamos con una amplísima bibliografía en torno a las relaciones literarias entre España y Alemania en general, sobre la presencia y la fortuna de las obras cervantinas en Europa y en Alemania en particular. Recojo buena parte de esta bibliografía en PARADA (1997), y PARADA (1998); aunque necesitadas de actualización, me siguen pareciendo especialmente recomendables las visiones de con- 
Sobre suelo alemán crean nobles y letrados en el siglo XVII un gran número de sociedades que, guiándose por formas e intenciones de las academias italianas renacentistas, intentan favorecer un idioma que va a la zaga de las grandes lenguas que lo rodean, unas lenguas que, recién descritas y fijadas por medio de gramáticas y compendios, cuentan ya con un pasado renombrado y un presente de aspiraciones imperiales ${ }^{2}$. Modélica a este respecto es la Fruchtbringende Gesellschaft (Academia Fructificante), la primera de estas sociedades, creada el 24 de agosto de 1617 por iniciativa del 'Fürst' Ludwig von Anhalt-Köthen a imagen y semejanza de la Accademia della Crusca. Los objetivos de la sociedad quedan fijados en el Kurtzer Bericht/ der/ Fruchtbringenden/ Gesellschaft/ Zweck und Vor/haben./ -/ Gedruckt zu Coethen//Im Jahr/ /1622, esto es, en el Breve informe de la Fruchtbringende Gesellschaft, en el que se declara la razón y los propósitos de la misma:

Fuers ander/ daß man die Hochdeutsche Sprache in ihren rechten wesen und Standt/ ohne einmischung frembder außlendischer wort/ auffs moeglichste und thunlichste erhalte/ und sich so wohl der besten außsprache im reden/ alß der reinesten art im Schreiben und Reimen=dichten befleißigen ${ }^{3}$.

Por lo demás, que de la mejor manera posible se conserve el alto alemán en su modo y estado adecuado, sin mezcla de palabras extrañas y extranjeras, esforzándose tanto por la mejor pronunciación en el decir como por usar las formas más puras en el escribir y versificar ${ }^{4}$.

El uso del latín como lengua culta, del francés como lengua cortesana, la ausencia de una 'poderosa' tradición escrita, la fragmentación de la Iglesia de Roma, la falta de un núcleo político estable alemán, amplio y aglutinador, el menoscabo y acoso que en Europa sufren las formas y expresiones populares, todo ello va a motivar que el alemán en tanto lengua vea obstaculizado un desarrollo tanto más urgente en cuanto que la necesidad de que este se produzca se va sintiendo como imperiosa, lo cual va sin duda ligado a una suerte de conciencia 'patria' alemana que se incrementa y fortalece frente a lenguas y presencias que, al fin y al cabo, se perciben como extranjeras ${ }^{5}$.

junto que respecto a lo uno y lo otro ofrecen TIEMANN (1936), BRÜGGEMANN (1958) y SCHRAMm $\left(1962^{2}\right.$ ). Para el siglo XVII consúltese sobre todo SCHWEITZER (1954).

2 Del siglo XVI es también la primera gramática alemana, la de VALENTIN ICKELSAMER, Eine teutsche Grammatica, de 1534, el mismo año en que Lutero completa su Biblia de 1522 añadiendo la traducción del Antiguo Testamento.

3 LUDWIG VON ANHALT-KöTHEN, Kurtzer Bericht/ der/ Fruchtbringenden/ Gesellschaft/ Zweck und Vor-/haben./ -/ Gedruckt zu Coethen//Im Jahr/ /1622, por ScHöNE $\left(1988^{3}\right)$, pp. 38-40, cita en p. 39.

4 Salvo que se indique lo contrario, las traducciones son mías (A. P.).

5 Un breve, reciente e interesante análisis de la evolución de las lenguas euro- 
Ahora bien, la historia de las literaturas nacionales europeas, o una simple mirada sobre las fuentes de cualesquiera obras señeras de nuestra cultura occidental, nos enseña que aun en los tiempos de mayor aislamiento político, en épocas de crueles guerras, el diálogo, y con ello, el intercambio cultural no cesa, permanece siempre vivo. Todavía hoy desconocemos muchos de los caminos por los que el conocimiento atravesaba las fronteras, y constatamos asombrados la rapidísima propagación de obras que, apenas publicadas en su país de origen, aparecen ya traducidas, citadas, comentadas a miles de kilómetros de las imprentas de las que salieron. Si escritas en latín, los cultos y académicos podían leerlas; si escritas en el idioma vernáculo, caso de las literaturas nacionales, el conocimiento de idiomas distintos al propio o las traducciones son el modo de acceder a la lectura de las mismas.

La suerte de muchas obras, y el rumbo mismo que a partir de estas toman otras, y con ellas, las diversas literaturas de ámbitos geográfico-culturales más o menos acotados, queda determinada en un ámbito supranacional por cuándo, qué y cómo se traduce. Y aunque parece existir desde un momento muy temprano una crítica literaria no oficial que selecciona para su divulgación aquello que también hoy valoramos, lo cierto es que ni se traduce todo a todos los idiomas ni los efectos que la aparición de una obra produce son los mismos en todos los países. En este sentido, la historia de las literaturas europeas como hecho de traducción y adaptación está todavía por escribir ${ }^{6}$.

En la feria de libros de otoño de 1621 se anunciaba la publicación inminente de la traducción alemana del Quijote bajo el título de: Ritterliche Thaten des wunderseltzamen Abenthewers Don Kichote de la Mantscha, zu teutsch/Juncker Zwarckflachens aus Fleckenland/ aus Spanischer Sprach in die Teutsche versetzet (Cothenis Anhaltinorum). Sin embargo, esta traducción, cuyo mismo título podría dar lugar a amplias disquisiciones sobre la práctica de la traducción literaria en el siglo XVII, ya que el caballero pasa a "Junker“ y se intenta traducir literalmente no solo el famoso 'quijote' sino incluso el topónimo, que se convierte en el 'País de las Manchas',

\footnotetext{
peas en relación con la evolución política y el surgimiento de los estados nacionales lo ofrece ahora BAGGIONI (1997). Para lo que aquí se trata pueden consultarse las pp. 146-150. Lograda la unificación lingüística alemana en el siglo XVIII, „Le paradoxe sera donc de voir une langue nationale (ou plutôt ses locuteurs, car les langues ne sont que des abstractions sourdes et muettes) à la recherche d'un État unitaire lorsque d'autres États (Italie, Grèce et plus tard Roumanie, Bulgarie) auront le plus grand mal à faire vivere dans les faits une langue nationale pour devenir des États-nations modernes." (BAGGIONI, p. 150).

6 Interesante como intento y modelo de una posible historia de la traducción es Delisle/Woodsworth (1995).
} 
no se publicó hasta 1648 , probablemente retocada. El título era Don Kichote de la Mancha. Das ist: Juncker Harnisch aus Fleckenland/ Aus Hispanischer Sprach in hochteutsche übersetzt. Franckfurt/ In Verlegung Thomae Matthiae Götzen, 1648, figurando como segunda portada la leyenda de: Die Abentheurliche Geschichte des Scharpffsinigen Lehns- und Rittersassen/Juncker Harnisches aus Fleckenland/ Auß dem Spanischen ins hochteutsche versetzt Durch Pahsch Basteln von der Sohle. Hoff Geißmar/ Gedruckt bey Salomon Schadewitz ${ }^{7}$.

Desde el punto de vista de la historia de la traducción y de la traductología, la introducción de Joachim Caesar, alias Pahsch Bastel von der Sohle, a su versión del Quijote es un verdadero hallazgo y legado, pues además de estar dedicada por entero a cuestiones de traducción, la claridad de ideas, el rigor y el orden con que estas se exponen revelan una conciencia de la labor de la traducción y de las distintas implicaciones socio-culturales que la tarea conlleva realmente llamativa para la época.

Obsérvese si no cómo estructura von der Sohle su introducción y lo que cabe deducir desde un punto de vista teórico-metodológico de sus palabras ${ }^{8}$ :

7 Sobre esta primera traducción contamos con muchos trabajos, que giran especialmente en torno a la identidad de ese primer traductor oculto tras el (probable) seudónimo de Pahsch Bastel von der Sohle. El estado actual de la cuestión, que, si no se me escapa nada, se corresponde todavía con las averiguaciones que Tiemann presentó en su artículo de 1933 (Tiemann, 1933) es que Pahsch Bastel von der Sohle es seudónimo de un tal Joachim Caesar, políglota de mundo, poeta y filósofo, oriundo de Halle. Se desconoce su fecha de nacimiento y muerte, pero por una pista textual se sabe que tuvo que nacer antes de 1588 .

Remito, pues, a la bibliografía sobre esta primera traducción para las cuestiones generales y como trasfondo de lo que a continuación se expondrá arriba en el texto.

8 Consúltese el 'Nachwort' (Epílogo) de TIEMANN a su edición facsimilar de 1928 (TIEMANN, 1928), que uso aquí junto con la edición original de 1669 , donde en primera portada figura: Don Kichote de la Mantscha. Das ist: Juncker harnisch aus Fleckenland. Aus hispanischer Sprach in hochteutsche übersetzt, Franckfurt, In Verlegung Thomae Matthiae Gotzeir, con un grabado a la izquierda retrato de Don Quijote y, al fondo, en segundo plano, Sancho, e interpuesto a la derecha entre título y editor el lema (frecuente para la época): kauff mich: Und liss mich; Reuts dich: So friss mich.; Odr ich Bezahl dich, esto es: cómprame: y léeme; Te arrepientes: devórame; $O$ yo te pago (sic, quizá con el sentido de, en caso contrario, yo te pago a ti; pero no queda claro); en la segunda portada figura: Die Abentheurliche Geschichte des scharpfsinnigen Lehns- und Rittersassen Juncker Harnisches aus Fleckenland, aus dem Spanischen ins hochteutsche versetzt durch Pahsch Basteln von der Sohle, Franckfurt, Gedurch bey Blasium Ilssnern (?), Im Jahr 1669. Esta edición lleva una introducción dedicada al lector que es la misma que la que figura en la edición de 1648. Consúltese también IN DER SMITTEN (1986). A mí me interesan ahora sobre todo las implicaciones socio-culturales e históricas de planteamientos y procedimientos de traducción. Creo que habría que replantearse desde este punto de vista la recepción de la literatura española del Siglo de Oro en Europa en 
1. Inicia el traductor su discurso declarándose, con toda modestia, hombre 'viajado', que conoce, por lo tanto, otros países y otras culturas; de esta forma demuestra su competencia cultural.

2. Señala a continuación a aquellos que le motivaron a llevar adelante la traducción, tarea que él ya había iniciado: „empujado por gentes de alto estado y gentes de entre los comunes“ („von hohes und mittelmässigs Stands Orten bin angestrengt worden “, p. $9^{9}$ ); su labor responde, pues, a una demanda social.

3. Alaba la obra que se propone traducir como uno de los mejores „Schlachtbücher" ${ }^{10}$, libro de caballerías, destacando que esta obtuvo mayor y mejor acogida fuera del propio país del autor, lo que el que ahora la vierte al alemán tuvo ocasión de comprobar personalmente y de primera mano: „pues pude notar que se estimaba mucho más entre otros pueblos, cualesquiera que fuesen, que en su propio país“ („ich doch in seinem GeburtsLande bey weitem dessen Würde nicht so hoch / als bey jedwedern andern Völckern / hab vermercken können." (p. 10). De este modo, el traductor ofrece también una pequeña historia de la recepción.

4. A continuación menciona y valora las traducciones que vio y conoció, a saber: la inglesa, que es la de Thomas Shelton, Londres 1612, y la francesa, que César Oudin y François de Rosset publicaron respectivamente en 1614 (primera parte) y 1618 (segunda parte). La primera, que ya no tiene a mano, debe de considerarse buena, más por lo que decían los ingleses, que por el dominio, escaso, que von der Sohle reconoce tener del inglés. La segunda, que usa para cotejar pasajes, aunque meritoria, es demasiado literal, se pega en exceso a la letra.

general, en Alemania en particular, y hacer una especie de historia social de las traducciones de literatura española en Europa. Piénsese por ejemplo en la decisiva, decidida e interesada tarea de traductor de Aegidius Albertinus, propagador de una novela picaresca española que en buena parte difiere del original que se conoce en la Península Ibérica.

9 Cito siempre por la edición de Tiemann de 1928, que no presente problemas de legibilidad.

10 Creo que no ha de tomarse esta referencia, motivo de muchas controversias, al pie de la letra. No es este el lugar de discutirlo, pero parece poco probable que von der Sohle, que conoce muy bien 'su obra', y por lo tanto también las severas derrotas que sufre Don Quijote, celebre el libro como un remedo especialmente conseguido de la serie de los Amadises, cuya suerte europea puede consultarse en el magnífico estudio de WedDIGE (1975). 
A la vez que el traductor practica, pues, crítica de la traducción nos descubre parte de su propia metodología.

5. Ofrece ahora sus criterios de traducción: „que toda traducción bien hecha debe ser de tal manera que la obra que se traduce parezca como si hubiera sido escrita originariamente en la lengua materna del traductor" (daß jedwede rechtmässige Dolmetschung also beschaffen seyn solle / samb wer das Werck / so darinnen gedolmetscht wird / uhrsprünglich in des Dolmetschen Muttersprach beschrieben." (p. 11)) Congruente con la crítica dirigida a la traducción francesa, von der Sohle toma a griegos y romanos como modelo: „los cuales acostumbraban a fijarse más en el sentido y la intención, que en las palabras y en el texto“ („welche gleichmässig mehr off Verstand und Meinung / als Worte und Text / ihr absehen gehabt.“ (p. 12) Así, von der Sohle parece concertar incluso con las más modernas teorías funcionalistas de la traducción, que priman función e intención por encima del texto materia lingüística. De ahí también que se imponga y sea necesario un sentido pragmático de la tarea, traducir implica tomar decisiones „y no perder demasiado tiempo devanándose una y otra vez los sesos sobre las palabras y el sentido" (,nicht allzuviel zeit verschertzen und aller Orte in ergrübelung Wörter oder Verstands so gar aberglaubisch seyn", p. 13) ${ }^{11}$. En este punto, von der Sohle se revela como un teórico de la traducción.

11 Creo que es a estas cuestiones de traducción a lo que se refieren los versos con los que von der Sohle subraya su opinión: „Ein Geck ist / der den Kopff uber Mährlein ihm zerbricht: // Die Arbeit thöricht ist / die man uff Thorheit richt" (p. 13) („Es necio quien sobre fantasmagorías se mata la cabeza: // necio el trabajo que a necedades se aplica.") También el contexto parece confirmar esta hipótesis, pues von der Sohle enlaza con toda naturalidad con el párrafo siguiente, en el que sigue tratando cuestiones de traducción: „Hierumb ich mir dann die Freyheit genommen / zuweilen so wol ein Spanisch Wort mit zwey Teutschen zugeben...” (p. 13) („Por lo cual me tomé la libertad / de reproducir en ocasiones una palabra española con dos alemanas...").

Dirimir esta cuestión no es asunto baladí, pues en la introducción que Hoffmeister antepone a su edición de la Historia von Isaac Winckelfelder und Jobst von der Schneidt, es decir, Rinconete y Cortadillo (HoFFMEISTER, 1983) se traen a colación estos versos como testimonio de la supuesta opinión negativa que, por „bufonada“, le merecería a von der Sohle la obra que traduce; concluye Hoffmeister: „Diese Äußerung darf als repräsentativ für die Einstellung der Gelehrten des Barockjahrhunderts gelten, die den Don Quijote unter die satirischen Schelmenromane einreihten.“ (HOFFMEISTER, p. 13) („Esta opinión puede considerarse representativa de la que le merecía la obra a los académicos o letrados del siglo del barroco, los cuales incluían el Don Quijote entre las novelas picarescas satíricas.") Hay que fijarse, sin embargo, en lo que Johann Rist, pastor (!) en Wedel, miembro también de la Fruchtbringende Gesellschaft, admirador incondicional de Bastel von 
6. Pasa después a hacer ya un análisis contrastivo entre la lengua española y alemana incidiendo en la distinta colocación del adjetivo, las diferencias en la formación de palabras y conceptos (formas analíticas versus sintéticas), en los préstamos, los registros, de los cuales destaca como interesante y dificultoso para la traducción la germanía, lo que él llama "gerigonça“, española, que exige el dominio de la correspondiente „Rohtwällische Schriftkunst“ etc. Con von der Sohle nos encontramos, pues, también ante un lingüista y filólogo contrastivo.

7. El traductor destaca algunas adaptaciones que se ha visto forzado a realizar, por tratarse de culturas distintas y ser desconocidas para el público destinatario de la traducción las realidades a las que ahí se alude; la grafía de los nombres se adoptó a la forma en que estos se pronuncian: Kichote, Mantzscha, Santzscho, Panssa, para que, dice, „el lector alemán sepa pronunciar las palabras españolas como las pronuncia el español [...] Pues en caso contrario, no le entendería el español ni ningún otro extranjero" cuando de la obra hablase. („hiermit der Teutsche Leser die Spanischen Wort recht außpreche / wie sie der Spanier außpricht [...] Dann ausser dem würd er von einem Spanier oder andern frembden nit verstanden werden“ (p. 17)) Así, el traductor se muestra consciente de ejercer de intermediario entre culturas.

8. Finalmente, el traductor se revela también como creador o autor, pues no solo omite aquello que le parece ajeno a la historia, como son cantos, versos, „largos cuentos e invenciones“ („lange Geschichte und Mährlein“ (p. 18)), aquello que se le antoja "langweilig“, aburrido, o de un humor excesivamente tosco, sino que varía también el número de capítulos y la estructuración de los episodios, práctica, por otro lado, bastante habitual para la época.

Hasta aquí, pues, la introducción de Pahsch Bastel von der Sohle.

Por encima de la competencia analítica, lingüística, cultural y literaria, destaca el sentido de comunidad del que von der Sohle hace

der Sohle, traductor también, de Tasso, tiene que decir sobre el original cervantino y la traducción de Caesar: „Que aparte de mí, todos los verdaderamente instruidos deseamos de corazón que en lengua alemana las restantes partes de este libro ingenioso salgan pronto pronto a la luz.“ („Welches scharffsinnigen Buches übrige Theile / daß Sie in Teutscher Sprache bald bald müchten herauf kommen / alle recht gelehrte / nebenst Mir / von hertzen wünschen." (cito por Tiemann, 1928, p. 418)

Considerando la introducción de von der Sohle en su conjunto, me parece de todo punto imposible llegar a las conclusiones que respecto a esta cuestión expone Hoffmeister. 
gala y la lealtad que a esta(s) profesa, pareja a la que demuestra respecto al texto que traduce. Si, por un lado, su tono se mantiene siempre objetivamente respetuoso hacia las culturas que menciona y que conoció, lo cual en un siglo de luchas y odios entre las naciones salta especialmente a la vista, von der Sohle no oculta que se siente parte de la sociedad - lingüística - para la que traduce y a la que trata de servir. El núcleo alrededor del cual lo uno y lo otro, y su labor misma, gira se resume en el concepto de comunicación.

Las premisas teóricas y metodológicas que afectan a su tarea de traductor están al servicio de una traducción que 'funcione' en la lengua de llegada sin alterar por ello significativamente, omisiones y alteraciones estructurales al margen, el texto original en lo que a la interpretación del mismo se refiere. Del mismo modo que busca que su traducción se encuadre de forma 'natural' en la cultura receptora, von der Sohle intenta conservar aquellos rasgos de la obra que le confieren un carácter transnacional, como lo son los nombres de los personajes. En la decisión de ofrecer cuasi una transcripción fonética de los mismos prima de nuevo el carácter comunicativo, pues no ha de verse entorpecido el diálogo $-\mathrm{y}$ puesto en evidencia quien el error comete- por una deficiente pronunciación, de modo que ni se reconozca la obra de la que se pretende hablar ${ }^{12}$.

Ahora bien. La puesta en práctica de un planteamiento 'dialógico' aplicado a la traducción, inherente al mismo proceso de traducción desde el momento en que se coteja el texto original con otras traducciones ya existentes y estas entre sí, no es sólo cuestión de voluntad o talante, sino también, y quizás primordialmente, de competencia. En su manifestación ideal, esta competencia alcanza el grado de 'biculturalidad', que implica y posibilita un concepto de tan difícil definición y precisión como es el de 'sensibilidad' lingüística y cultural, que podríamos definir como capacidad intituitivoracional extremadamente desarrollada para captar, interpretar y, dado el caso, expresar matices. Que Bastel von der Sohle, que contempla el Quijote como un fenómeno ya transnacional, no llega, a pesar de un dominio (pasivo) del castellano de la época que ha de calificarse sin duda de magnífico, a esos extremos nos lo demuestran los diversos errores que comete a la hora de interpretar el texto original (TIEMANN, 1928, p. 412).

\footnotetext{
12 Trasluce aquí una intención educativa de formar hombres de mundo como más tarde reaparecerá en Christian Thomasius, que en el semestre de invierno de $1667 / 68$ trata el Oráculo manual y arte de prudencia de Gracián buscando que sus estudiantes se convirtiesen en "beaux esprits, hommes de bon gout et galands"; THOMASIUS (1970).
} 
Con todo, lo verdaderamente importante es que Pahsch Bastel von der Sohle, traductor del Quijote, hace gala en su introducción de una capacidad de abstracción y un sentido histórico de su tarea de traductor que trasciende momento y obra y señala, tanto desde un punto de vista teórico-metodológico como en el plano práctico, hacia el futuro. Su capacidad de valorar el hecho literario-cultural desde el punto de vista meramente formal o 'técnico', así como de comprender la obra inserta en los distintos contextos nacionales, coadyuvan de forma esencial a ello.

De lo uno y lo otro, de todo ello, no sólo ofrecen testimonio fehaciente las decisiones de traducción que adopta, sino también, y de forma concluyente, la influencia que su traducción, de, recuérdese, sólo veintitrés capítulos, ejerció todavía en versiones posteriores, pues tanto la versión de Friedrich Justin Bertuch, también parcial, del Quijote, de 1775, como la de Dietrich Wilhelm Soltau, de 1800 , e incluso la renombrada de Ludwig Braunfels, de 1873, remiten, agradecidas, al trabajo realizado cien, doscientos años atrás por Pahsch Bastel von der Sohle ${ }^{13}$.

Cabe concluir, pues, que el primer traductor del Quijote respondía a la perfección a las exigencias que se le pudieran plantear a un 'mediador cultural': amplitud de miras, competencia profesional, rigor y entusiasmo por su tarea. En este sentido, tanto coetáneos como la posteridad tuvieron que lamentar que Joachim Caesar, si es que de él se trataba, no concluyera su trabajo de interpretar, traducir y, al fin y al cabo, comunicar el Quijote.

ARTURO PARADA

Universidade de Vigo

\section{REFERENCIAS BIBLIOGRÁFICAS}

BAgGioni, Daniel (1997): Langues et nations en Europe. Éditions Payot \& Rivages, París.

BRÜGgemann, WeRnER (1958): Cervantes und die Figur des Don Quijote in Kunstanschauung und Dichtung der deutschen Romantik. Spanische Forschungen der Görresgesellschat, Aschendorff.

Cervantes SaAvedra, Miguel De (1669): Don Kichote de la Mantscha. Das ist: Juncker Harnisch aus Fleckenland / Aus hispanischer Sprach in hochteutsche übersetzt. Franckfurt, In Verlegung Thomae Matthiae Gotzeir.

Delisle, JEAN/WoOdsWORTH, JUDITH (1995): Translators trough history. John Benjamins, Amsterdam-Filadelfia.

13 Sobre esto puede verse KRONACHER (1923) y las correspondientes introducciones de Soltau y Braunfels a sus traducciones de 1837 y 1873 respectivamente. 
HofFMeister, Gerhart (1983), Introducción a Ulenhart, Niclaus: Historia von Isaac Winckelfelder und Jobst von der Schneid. Reclam, Leipzig, pp. 5-35.

IN DER SMITTEN, THeo (1986): Don Quixote (der "richtige" und der "falsche") und sieben deutsche Leser. Rezeptionsästhetische leseaktorientierte vergleichende Analysen an spanischen Ur-Quixote-Ausgaben von 1604/1605 bis 1615 und sechs deutschen Übersetzungen von 1648 bis 1883 (2 tomos.). [Diss.] Peter Lang Verlag, Berna.

KRONACHER, BETTINA (1924): Bertuchs Don Quijote-Übersetzung unter Einbeziehung der ihm nächstfolgenden Übersetzungen von Tieck und Soltau. [Tesis Doctoral] Múnich.

Parada, Arturo (1997): Offene literarische Welten gegen geschlossene Denkmodelle und Sozialsysteme: Don Quijote und Anton Reiser. Vervuert, Francfort am Main.

- (1998): „Die 'semínimas literarias' von Cervantes bis Blanckenburg: Aufriß eines europäischen Stranges“, en Grupo de Investigación Filología Alemana (edts.): Tradición e innovación en los estudios de lengua, literatura y cultura alemanas en España. Sevilla, pp. 251-258.

SCHÖNE, AlBRECht (19883) (edit.): Das Zeitalter des Barock. Texte und Zeugnisse. C. H. Beck, Múnich.

SCHRAMM, EDMUND (1962²): „Die Einwirkung der spanischen Literatur auf die deutsche“, en Stammler, Wolfgang (edt.): Deutsche Philologie im Aufriss. Erich Schmidt Verlag, tomo III, columnas 147-200.

SCHWEITZER, CHRISTOPH Eugen (1954): Spanien in der deutschen Literatur des 17 Jahrhunderts. [Tesis Doctoral] Yale.

THOMASIUS, CHRISTIAN (1667/1970): „Christian Thomas eroeffnet der Studirenden Jugend zu Leipzig in einem Discours Welcher Gestalt man denen Frantzosen in gemeinem Leben und Wandel nachahmen solle? ein Collegium über des Gratians Grund=Reguln/ Vernuenfftig/ klug und artig zu leben“, en THOMASIUS, CHRISTIAN: Deutsche Schriften (edit. Peter von Düffel). Reclam, Stuttgart, pp. 8-49.

Tiemann, Hermann (1928): Epílogo a la nueva edición de: Don Kichote de la Mantzscha, Das ist: Juncker Harnisch aus Fleckenland. Friederichsen, de Gruyter \& Co., Hamburgo, pp. 401-424.

- (1933): „Der deutsche Don Kichote von 1648 und der Übersetzer Aeschacius Major", en Zeitschrift für deutsche Philologie, 58, pp. 232-265, y en TiEmanN, HERMANN (sin año: 1974): Essays, Vorträge und Aufsätze aus vier Jahrzehnten. Maximilian Gesellschaft in Hamburg, Hamburgo, pp. 93-126.

- (1936): Das spanische Schrifttum in Deutschland von der Renaissance bis zur Romantik. Ibero-Amerikanische Studien 6, Hamburgo.

Weddige, Hilkert (1975): Die Historien von Amadis auß Frankreich. Dokumentarische Grundlegung zur Entstehung und Rezeption. Franz Steiner Verlag, Wiesbaden. 